\title{
What People Say: Application of Rural Definitions and Descriptors of Community in Ingersoll, Ontario
}

Neil Stoop

University of Guelph 


\section{Back Drop to Research}

- How we define and describe communities impacts the individuals who live there.

- A definition of rural, and what constitutes a rural community has been the focus of academic and political debate.

- While the academic debate exists, it is often not reconciled with the lay discourse. 


\section{Key Research Questions}

- How do residents of Ingersoll Ontario describe their community?

- How does the lay discourse of rural differ from the academic and political discourse, as found in Ingersoll Ontario? 


\section{How is rural defined?}

- Descriptive definitions

- Rural is defined by their socio-spatial characteristics

- Socio-cultural definitions

- Rural is defined by the way people act and behave in rural areas

- Rural as a locality

- Rural is defined by the existence of structures operating at a local level

- Social Representations of rural

- Rural is defined with input from the lay discourse, and how people view and describe rural 


\section{How is rural defined?}

- OMAFRA RED Program

- <100 o00 people and < 100 people/km²

- Northern Rural Remote Recruitment and Retention Program

- an index scoring a communities population, density, health services and travel times to referral centers

- Census Rural

- <1000 people, or < 400 people/ $\mathrm{km}^{2}$ 


\section{Ingersoll, Ontario}

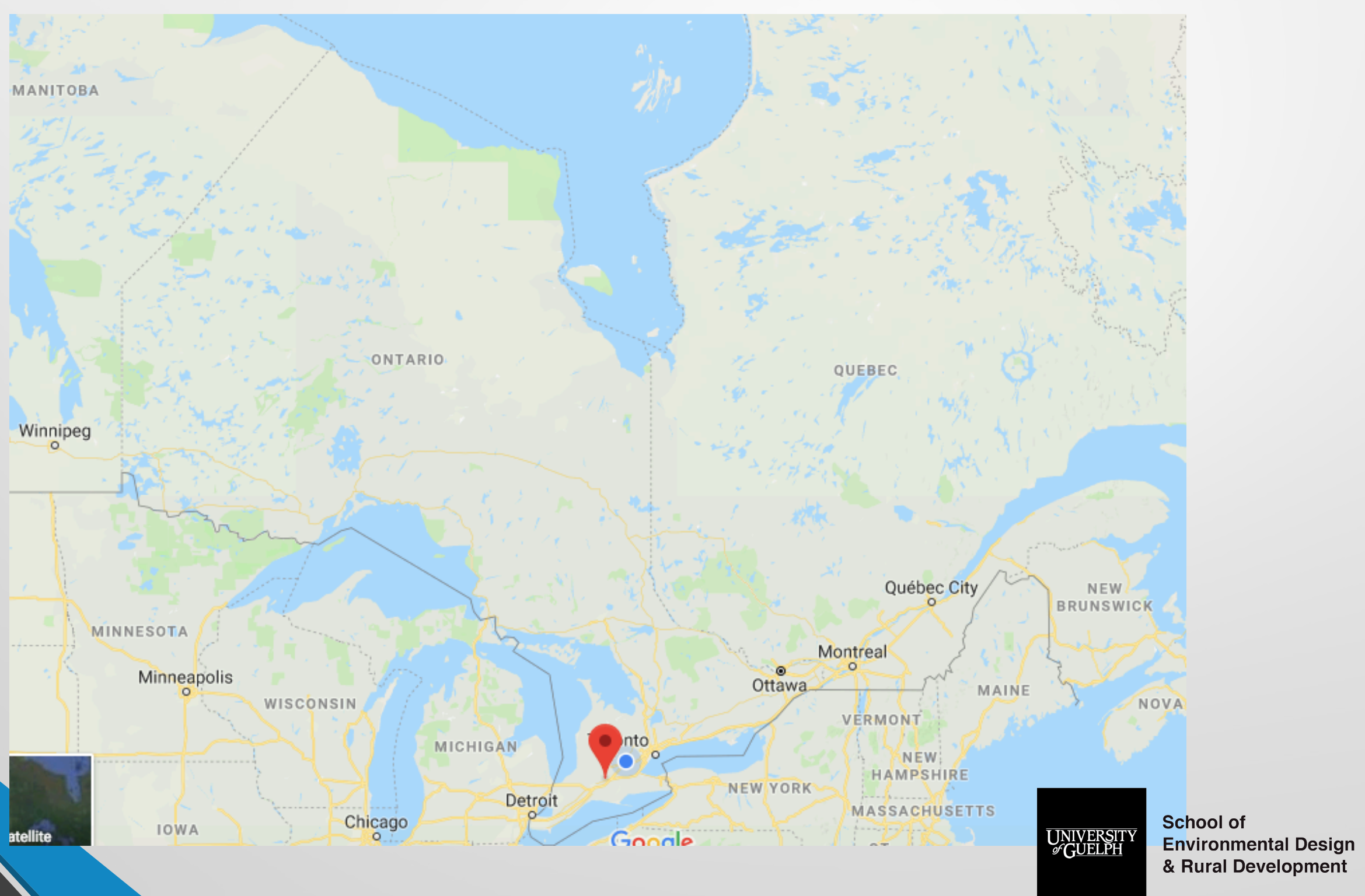




\section{Ingersoll, Ontario}

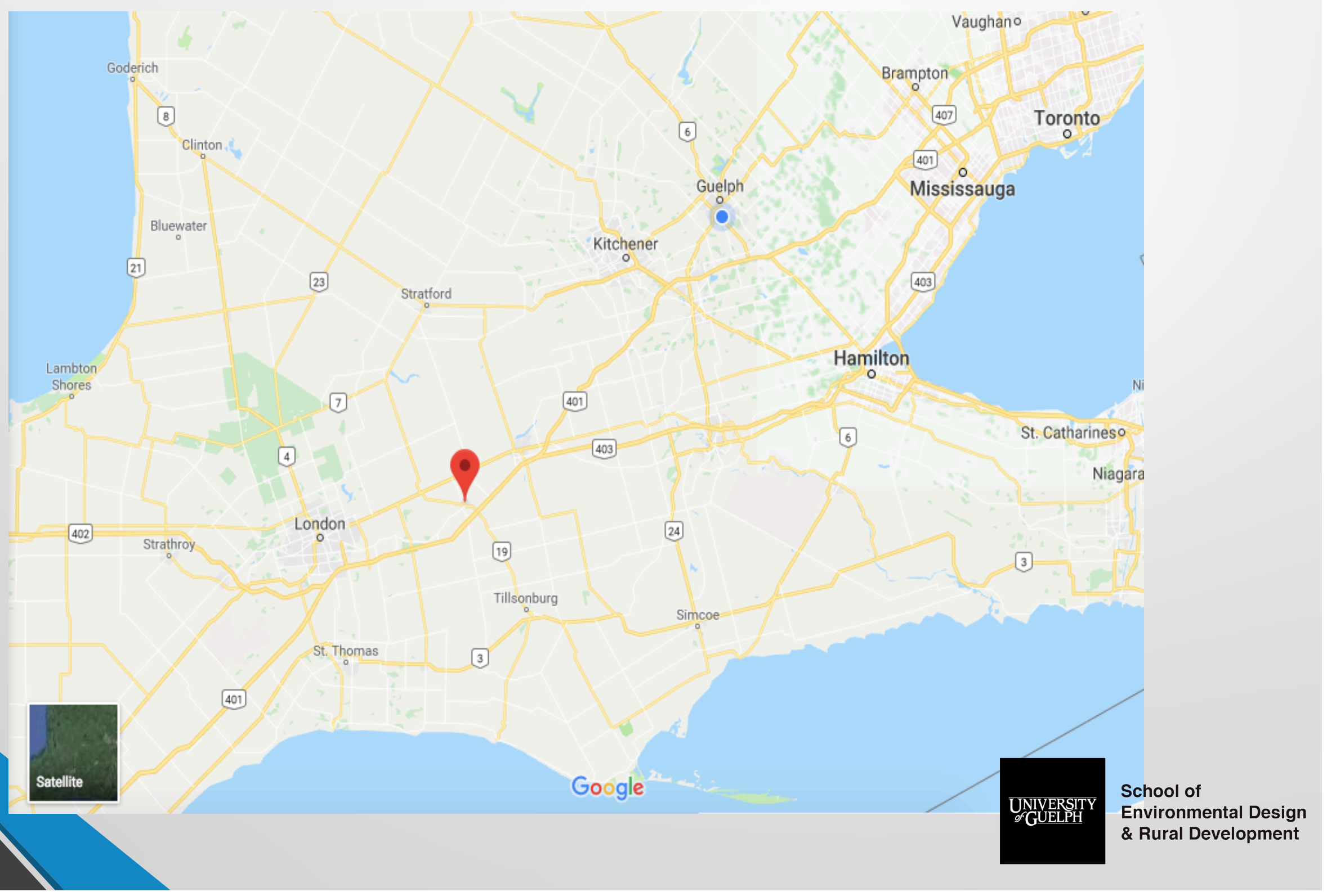




\section{Ingersoll, Ontario}

- Population: 12757 (5\% increase from 2011 to 2016)

- Population Density: 1000 people per $\mathrm{km}^{2}$

Top Industries (NAICS)

Manufacturing

Health care and social assistance

Retail trade

\section{Number Employed (2011)}

740

715 
What are people in Ingersoll saying? 


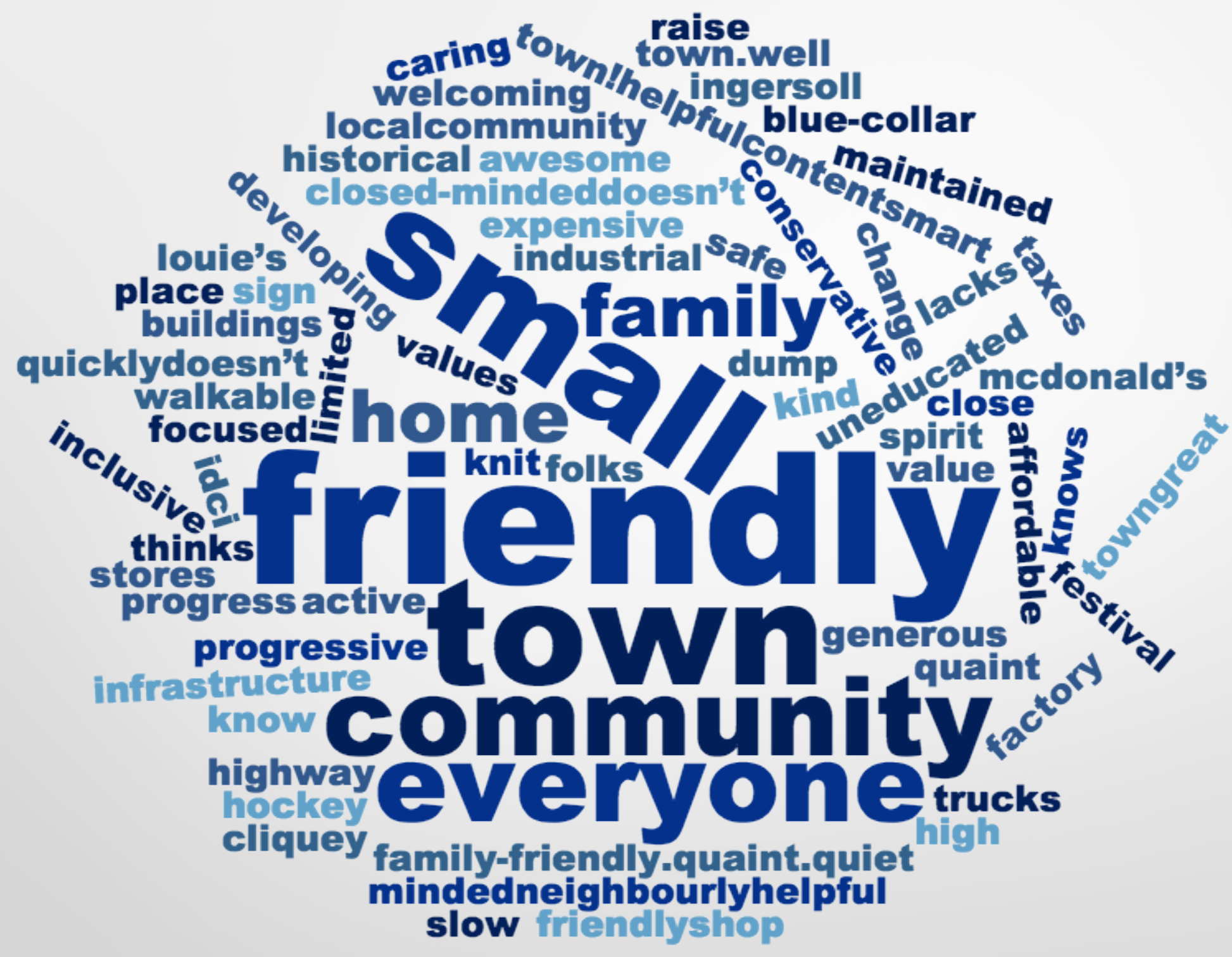




\section{Is Ingersoll rural?}

16

14

12

10

8

6

4

2

0

Yes

No 


\section{What are the implications for rural Ontario?}

- Who is and is not included in rural Ontario?

- The way communities are described and classified impacts the way they are viewed by both residents, and outsiders.

- Altering definitions and description affect how communities are impacted by policy and programs. 


\section{Moving Forward}

- The way individuals and organizations describe communities differs and it is necessary to be aware of these differences.

- When using a term such as 'rural' it is important to define what it means in the particular context.

- Academics and policy makers alike need to be aware of the 'lay discourse' that surrounds communities. 


\section{Contact Information}

\section{Neil Stoop}

nstoop@uoguelph.ca 
Thank-you 